\title{
Coping with Stress in Adolescents with Type 1 Diabetes and THEIR MOTHERS
}

\author{
E. Pisula and Z. Czaplinska \\ Faculty of Psychology, Warsaw University, Warsaw, Poland
}

\begin{abstract}
Objectives: Coping with stress plays a vital role in the adjustment of adolescents with diabetes. The majority of studies in this area leave out the control group, limiting their power to make inferences about specificity $v s$. similarity of coping strategies used by these adolescents. The aims of this study were: (1) To compare coping strategies in adolescents with diabetes and healthy adolescents; (2) To compare coping strategies in girls and boys with diabetes; (3) To determine whether there is a relationship between adolescents' coping strategies and their mothers' coping styles.

Material and methods: Adolescents (12-17 years old) with Type 1 diabetes $(\mathrm{n}=51)$ were compared with a control group of healthy secondary school students (n $=56$ ) by means of a self-reported questionnaire measuring coping strategies (Adolescence Coping Checklist). Mothers of these adolescents $(n=107)$ completed the Coping Inventory for Stressful Situations, measuring 3 coping styles.

Results: Diabetic adolescents used the 'seek professional help' strategy more often than their healthy peers. The girls with diabetes reported using the 'investing in close friends' strategy more often than boys, while in the control group girls were also more likely to use 'seeking social support', 'seeking spiritual support', and 'relaxing diversions' strategies. Mothers' emotion-oriented coping style predicted focus-oriented coping in adolescents with diabetes. In the non-diabetic group, mothers' task-oriented coping predicted seeking professional help, while mothers' avoidanceoriented coping predicted seeking spiritual support.

Conclusions: The results demonstrated that: (1) the only differences in terms of coping strategies in adolescents with diabetes and healthy adolescents were found in seeking professional help; (2) gender differences in coping with stress were significantly smaller in adolescents with diabetes than in healthy adolescents, (3) mothers' coping styles were predictors of coping strategies in adolescents, albeit there were differences in that respect between adolescents with diabetes and healthy adolescents.
\end{abstract}

Key words: adolescents, Type 1 diabetes, coping strategies, coping styles, mothers

\section{INTRODUCTION}

Any chronic illness, such as Type 1 diabetes, can be a source of significant stress for adolescents (Beveridge et al. 2006; Huus and Enskär 2007). This stress represents an additional burden in this already difficult period, during which young people encounter numerous challenges in their relationships with parents and peers, at school, or related to self (Hema et al. 2009). Diabetes-related stress has been shown to be an important predictor in the development of maladjustment in adolescents (Malik and Koot 2009). There is also a relationship between coping styles and quality of life and metabolic control in individuals with this condition (Graue et al. 2004). In this light, the understanding of factors that influence coping with stress in adolescents with diabetes seems to be particularly important. Such information may be useful in the planning of measures aimed at developing the adaptive abilities which play an important role in managing health problems.

The results of research on coping strategies in adolescents with diabetes are inconclusive. Some authors claimed that there were no differences between this group and healthy peers (Hema et al. 2009), while others found such differences (e.g. Olson 1993), as well as differences in coping between individuals with diabetes and adolescents with other health conditions (Petersen et al. 2006). The amount of data on gender differences in coping strategies in adolescents with diabetes is also limited, although reports on other groups of adolescents suggest that there should be clear differences at this stage of development (e.g., Frydenberg and Lewis 2009).

Diabetes in adolescents is particularly involving for their families (Seiffge-Krenke 2001). Parents are an important source of support for teenagers, and they influence the development of their adaptive skills, including the ability to cope with diabetes-related challenges (Olsen et al. 2008). The level of stress in mothers has been shown to affect the development of adaptive skills in youth with Type 1 diabetes and to be associated with depressive symptomatology (Mullins et al. 2004). Having the mother help them cope with problems has a positive effect on adolescents' adaptation.

The specific aims of this study were to assess: (1) whether there were any differences in the use of coping strategies between adolescents with diabetes and healthy adolescents; (2) whether there were any differences between coping strategies used by girls and boys in these groups; (3) whether there was a relationship between coping strategies used by youth and coping styles in mothers; and (4) whether there were any differences in stressors reported by adolescents with diabetes and healthy adolescents. 


\section{Material AND Methods}

The research has been conducted in accordance with local legal and ethical regulations for scientific research in Poland. A written consent of each participant was collected. No incentive was offered for participation in the study.

Families of adolescents with diabetes were contacted at diabetes clinics and hospitals. Adolescents were contacted once parents' consent for their child's participation had been obtained. After they consented, mothers and adolescents were given a set of questionnaires. Participants returned completed questionnaires to the experimenter. Families of healthy adolescents were contacted through schools and a scouting troop.

\section{PARTICIPANTS}

There were 214 participants in the study: 107 adolescents, including 51 with Type 1 diabetes and 56 healthy ones, and 107 mothers of the participating adolescents. The age range was $12-17$ years. In the diabetic group (mean age $14.0 \pm 1.5 \mathrm{SD}$ years), there were 26 girls and 25 boys. In the healthy group (mean age $14.6 \pm 1.6$ years), there were 36 girls and 20 boys. The mean age of the mothers of adolescents with diabetes was $41.8 \pm 6.0$ and of the mothers of the healthy youth was $42.7 \pm 5.3$. Duration of disease was approximately 1 year in 16 participants, 2-5 years in 17 participants, 6-10 years in 14 participants, and 11 or more in 4 participants. Selection criteria for the control group were age and declared health status (absence of chronic diseases). There were no differences between the study groups in terms of the distribution of sex and age of participants and their families' financial standing. There were differences, however, in terms of mothers' education (more mothers of adolescents with diabetes had primary or vocational education than mothers of healthy adolescents, $\mathrm{Chi}^{2}=4.46$, $\mathrm{P}<0.05$ ) and professional activity (mothers of diabetic adolescents were unemployed more frequently than their counterparts, $\left.\mathrm{Chi}^{2}=11.64, \mathrm{P}<0.01\right)$.

\section{Psychometric TOOLS}

Adolescents' coping strategies were assessed using the Adolescence Coping Checklist (Frydenberg and Lewis, 1990), adapted for Poland by Sikora and Pisula (2002). The questionnaire is aimed at individuals aged 11-19. It consists of 48 items, with 13 scales identified through factor analysis, representing the following coping strategies:

1. Ignore the Problem - consciously blocking out the problem and giving up (e.g. ,,Shut myself off from the problem so that I can avoid it").

2. Seeking Social Support - sharing the problem with others and involving them in the solving process (e.g. „Seek encouragement from others”).

3. Focus on Solving the Problem - working through the problem systematically, learning more about the problem and considering alternative solutions (e.g. „Analyse and think about the problem: think of a different solution").
4. Work Hard and Achieve - commitment, diligence and being driven to succeed (e.g. „Achieve well in what I'm doing").

5. Focus on Leisure Activities - looking to have a good time (e.g. „Go out with friends and have a good time and forget about troubles").

6. Focus on the Positive - making cognitive efforts to change the perception of the stressor (e.g. „Look on the bright side of things and think of all that is good").

7. Seek Spiritual Support - coping through prayer and religious devotion (e.g. „Pray for help and guidance so that everything will be alright").

8. Seek Professional Help - seeking assistance from a qualified adult (e.g. „Ask for advice from a qualified person").

9. Worry - worrying about the course of events (e.g. „Worry a lot about mu future”).

10. Investing in Close Friends - tightening relationships and making new friends (e.g. „Make approaches to a guy or girl").

11. Seek to Belong - seeing others as supportive and appreciating their importance (e.g. „Make a good impression on others who matter to me").

12. Seek Relaxing Diversions - undertaking calming activities: reading, painting, walking (e.g. „,Go for a walk").

13. Social action - engaging in group activities (e.g. „Organise an action or petition regarding my concern”).

Participants responded by marking the frequency of using each strategy on a 4-point scale (1 - "never"; 2 "rarely"; 3 - "often", and 4 - "always" or "almost always"). The reliability of the Polish version of the scale tested on a sample of 70 participants (Sikora and Pisula 2002) ranges for various subscales from $r=$ 0.78 to $r=0.44$.

The Coping Inventory for Stressful Situations CISS (Endler and Parker, 1990) adapted for Poland by Strelau et al. (2005) was used to assess coping styles in mothers. The tool consists of 48 items making up three scales:

- task-oriented coping - making efforts aimed at solving the problem (e.g., "Work to understand the situation"),

emotion-oriented coping - self-oriented emotional reactions, focusing activity on the reduction of emotional tension caused by the stressor (e.g., "Become very upset"),

- avoidance-oriented coping-avoiding a stressful situation in one of two ways: avoidance by social diversion (e. g., "Visiting or phoning a friend") and avoidance by distraction (e. g. "Go out for a snack or meal").

Participants responded on a 5-point Likert scale (from 1 - not at all to 5 - very much). The validity and reliability of the Polish version of CISS is high (Strelau et al. 2005).

In addition, mothers completed a demographics survey containing questions about their age, education, family status, financial standing, professional activity, as well as their child's age and disease duration. In order to determine what stressors adolescents have encoun- 
tered over the two weeks preceding the study, participants completed a short survey containing an open question about the causes of stress in that period.

\section{STATISTICAL EVALUATION}

Data are reported as mean raw scores \pm SD. Rates of using coping with stress strategies in the group of adolescents with diabetes and controls, as well as coping styles in mothers of adolescents in both groups were compared using Student's t-test for independent samples. Student's t was also used to compare coping strategies in girls and boys in both groups of adolescents in the study. Furthermore, stepwise regression analysis was used to determine the predictors of adolescent coping strategies for each adolescent group separately.

\section{RESULTS}

\section{Coping Strategies in Diabetic and Healthy ADOLESCENTS}

The group of adolescents with Type 1 diabetes differed from the control group only in terms of seeking professional help (Table 1). Adolescents with diabetes used this strategy more frequently than their healthy counterparts.

\section{Coping Strategies in Girls and Boys}

Coping strategies used by girls and boys were compared in each group separately. Table 2 shows the results in both groups for those strategies where gender differences were found.

Mothers' Coping Styles and Level of Education as Predictors for Adolescents' Coping Strategies

The relationships between mothers' coping styles and their level of education and the coping strategies employed by adolescents were also analyzed. The results of regression analysis are shown in Table 3 for the group of adolescents with diabetes and in Table 4 for the control group.

\section{Types of STREsSors RePORTED By Diabetic AND Non-Diabetic Adolescents}

To analyze the stressors reported by diabetic and nondiabetic adolescents, the total number of reported stressors was calculated and four subject matter categories were identified: school-related stressors (e.g. tests, unfair grades, strict teachers), peer-related stressors (e.g. making new acquaintances, fear of embar-

Table 1. Data of principal variables.

\begin{tabular}{lcccc}
\hline \multicolumn{1}{c}{ Variables } & $\begin{array}{c}\text { Total } \\
(\mathrm{n}=107)\end{array}$ & $\begin{array}{c}\text { Diabetic } \\
(\mathrm{n}=51)\end{array}$ & $\begin{array}{c}\text { Healthy } \\
(\mathrm{n}=56)\end{array}$ & $\mathrm{P}<$ \\
\hline Ignore the Problem & $16.4 \pm 3.2$ & $16.3 \pm 3.5$ & $16.5 \pm 3.0$ & 0.79 \\
Seek Social Support & $12.0 \pm 3.0$ & $12.0 \pm 2.8$ & $12.0 \pm 3.2$ & 0.92 \\
Focus on Solving the Problem & $12.9 \pm 2.6$ & $12.9 \pm 2.6$ & $10.9 \pm 2.2$ & 0.10 \\
Work Hard and Achieve & $11.0 \pm 2.1$ & $11.1 \pm 2.1$ & $12.9 \pm 2.5$ & 0.62 \\
Focus on Leisure Activities & $13.0 \pm 2.6$ & $13.1 \pm 2.7$ & $9.4 \pm 2.3$ & 0.58 \\
Focus on the Positive & $9.4 \pm 2.4$ & $9.4 \pm 2.4$ & $5.6 \pm 2.2$ & 0.10 \\
Seek Spiritual Support & $5.7 \pm 2.2$ & $5.8 \pm 2.1$ & $3.6 \pm 1.3$ & 0.65 \\
Seek Professional Help & $3.8 \pm 1.2$ & $4.2 \pm 1.2$ & $5.0 \pm 1.7$ & $0.05 *$ \\
Worry & $4.9 \pm 1.6$ & $4.9 \pm 1.5$ & $5.1 \pm 1.8$ & 0.99 \\
Invest in Close Friends & $5.0 \pm 1.8$ & $5.0 \pm 1.8$ & $7.5 \pm 2.0$ & 0.92 \\
Seek to Belong & $7.6 \pm 1.8$ & $7.8 \pm 1.5$ & $6.4 \pm 2.1$ & 0.38 \\
Seek Relaxing Diversions & $6.7 \pm 2.0$ & $6.9 \pm 1.8$ & $3.7 \pm 1.0$ & 0.16 \\
Social action & $3.7 \pm 1.1$ & $3.8 \pm 1.2$ & $57.8 \pm 8.4$ & 0.69 \\
Task oriented coping & $56.9 \pm 7.4$ & $55.9 \pm 6.1$ & $43.1 \pm 8.8$ & 0.17 \\
Emotion oriented coping & $43.5 \pm 9.2$ & $44.0 \pm 9.6$ & $40.8 \pm 8.6$ & 0.58 \\
Avoidance oriented coping & $40.3 \pm 8.1$ & $39.8 \pm 7.6$ & $17.1 \pm 3.3$ & 0.53 \\
Avoidance by social diversion & $16.8 \pm 3.2$ & $16.4 \pm 3.1$ & $16.6 \pm 5.0$ & 0.25 \\
Avoidance by distraction & $16.7 \pm 4.8$ & $16.8 \pm 4.6$ & 0.86 \\
\hline
\end{tabular}

Table 2. Gender differences in using coping strategies in studied samples.

\begin{tabular}{lcccccc}
\hline Coping strategy & \multicolumn{3}{c}{ Adolescents with diabetes } & \multicolumn{3}{c}{ Healthy adolescents } \\
& Girls & Boys & $\mathrm{P}=$ & Girls & Boys & $\mathrm{P}=$ \\
\hline Seek Social Support & $12.2 \pm 3.0$ & $11.8 \pm 2.7$ & 0.63 & $12.9 \pm 3.0$ & $10.4 \pm 2.9$ & $\mathbf{0 . 0 0 4 *}$ \\
Seek Spiritual Support & $5.5 \pm 2.3$ & $6.0 \pm 2.0$ & 0.44 & $6.1 \pm 2.3$ & $4.7 \pm 1.7$ & $\mathbf{0 . 0 1 5 *}$ \\
Invest in Close Friends & $5.6 \pm 1.8$ & $4.4 \pm 1.8$ & $\mathbf{0 . 0 2 *}$ & $5.6 \pm 1.6$ & $4.1 \pm 1.8$ & $\mathbf{0 . 0 0 4 *}$ \\
Seek Relaxing Diversions & $7.3 \pm 1.9$ & $6.6 \pm 1.6$ & 0.18 & $7.2 \pm 1.9$ & $5.1 \pm 1.7$ & $\mathbf{0 . 0 0 1 *}$ \\
\hline
\end{tabular}


Table 3. Summary of hierarchical regression analysis for mothers' coping styles and level of education variables predicting adolescents' coping strategies in adolescents with diabetes sample $(\mathrm{n}=51)$.

\begin{tabular}{lccccc}
\hline Coping strategy & Predictor & $\mathrm{R}^{2}$ & Beta coefficient & $\mathrm{F}$ & $\mathrm{P}<$ \\
\hline Focus on Solving the Problem & Emotion-oriented coping & 0.11 & -0.34 & 6.56 & 0.05 \\
Social Action & Mother's level of education & 0.11 & 0.34 & 6.27 & 0.05 \\
\hline
\end{tabular}

Table 4. Summary of hierarchical regression analysis for mothers' coping styles and level of education variables predicting adolescents' coping strategies in healthy adolescents $(n=56)$.

\begin{tabular}{lccccc}
\hline Coping strategy & Predictor & $\mathrm{R}^{2}$ & Beta coefficient & $\mathrm{F}$ & $\mathrm{P}<$ \\
\hline Seek to Belong & Mother's level of education & 0.12 & -0.34 & 7.16 & 0.01 \\
Seek Professional Help & Task-oriented coping & 0.13 & 0.36 & 7.93 & 0.01 \\
Seek Spiritual Support & Avoidance by distraction & 0.14 & 0.37 & 8.68 & 0.01 \\
\hline
\end{tabular}

rassment), family-related stressors (conflicts with parents, fights with siblings), health-related stressors (illness, medical procedures), and other (death of a favorite pet, worrying about the future). The total number of stressors and numbers of stressors within each category were summed up for each participant. Analysis showed that the average number of stressors in the total sample was 1.34 (ranging from 1 to 6), while the average number of stressors within individual categories did not exceed 1 . There were no differences between the two groups in the total number of stressors. A difference was found in the number of stressors in the health category. These stressors were more prevalent in adolescents with diabetes than in healthy adolescents $(\mathrm{F}(1.106)=7.2 ; \mathrm{P}=0.008)$. No differences between the groups were found for the remaining stressor categories (school-, family- and peer-related, and others).

\section{Discussion}

Our results indicate that the rates of using specific coping strategies in diabetic and non-diabetic adolescents are fairly similar. The only exception was seeking professional help, a strategy which diabetic adolescents report using more often.

Lack of differences in the rates of using coping strategies is consistent with earlier reports (e.g. Hema et al. 2009). The fact that adolescents with diabetes sought professional help more often is likely to be related to the nature of stressors they experience. Among stressors reported by these adolescents, there were more health-related problems. There is no doubt that they experience more situations where they require professional medical assistance. This is likely to be the reason why they report seeking professional help more often.

With the procedure used in this study, it was possible to measure thirteen coping strategies most frequently used by adolescents (Frydenberg and Lewis 1990). It would be interesting to analyze strategies employed by them in the context of stressors directly associated with diabetes. As Schur et al. (1999) reported based on interviews with youth with diabetes: "the young people had developed sophisticated, interrelated self-protective strategies to manage intrapersonal and interpersonal threats from diabetes" (p. 223). Analogies between the groups in terms of coping strategies are probably related to the similarities between stressors reported by the participants. Groups differed only in terms of health-related stressors; there were no differences with respect to stressors related to family relations, peers, school, and other sources of stress. Similar results were obtained by Hema et al. (2010) who demonstrated that young diabetic patients experience a number of everyday miscellaneous stressors; and the stressors specifically related to diabetes and its management are not a major group among these pressures.

In the present study we also found fewer differences between girls and boys with diabetes than in the healthy group. The only difference was with respect to investing in friends: girls used this strategy more often than boys. In the control group, girls were also found to seek social and spiritual support, and engage in relaxing diversions more often. The direction of gender differences found in the present study is consistent with earlier reports (e.g. Pisula and Sikora 2008). The small extent of these differences in youth with diabetes may suggest that boys in this group resort more frequently to strategies typically employed by girls (Cf. Tab. 2). A possible explanation is that their health puts limits on their ability to use strategies typical for boys (e.g. physical activity, sports). These strategies have not been taken into account in the present study. Gender differences in coping with stress in young people with diabetes require further research.

Coping styles and mothers' level of education were predictors of two coping strategies among youth with diabetes and three in non-diabetes youth. Focus on solving the problem was negatively related in young people suffering from diabetes with emotional-oriented coping style in mothers. Thus, if the mother copes with stress by focusing on emotions, her adolescent child is less likely to use adaptively advantageous coping strategies that involve focusing on the problem at hand. Research results show that emotion-oriented coping is a predictor of parenting stress (Dabrowska and Pisula 2010). Thus, our results support the theory that proper educational and therapeutic support must be offered not only to adolescents with diabetes, but also to their parents. It has been well established that the child's diagnosis of diabetes is highly stressful for the mother, 
and that her stress may be reduced through appropriate educational measures (Hoff et al 2005).

In addition, in the group of adolescents with diabetes, mother's level of education was positively correlated with young people coping through social activities, which may indicate a more active way of dealing with stress by children of better educated mothers.

As for the non-diabetic group, adolescents whose mothers used more task-oriented coping reported more often that they dealt with problems by seeking professional help. Consulting a professional may be considered a rational way of approaching an issue. Mothers preferring the task-oriented coping style probably teach their children to look for alternative solutions, one of which is to ask a professional for help. On the other hand, individuals whose mothers tended to demonstrate avoidance by distraction were more likely to seek spiritual support. Thus, mother's avoidance of contact with stressors encourages the development in her teenage child of coping strategies involving prayer and falling back on supernatural powers, which by their nature have no direct effect on the circumstances that instigate that stress.

In conclusion, the results of the present study confirm the findings of other research projects in terms of small differentiation between adolescents with diabetes and their healthy peers in terms of coping strategies. The similarities in terms of coping with stress in girls and boys with diabetes revealed in our study may serve as a starting point for further research, which should also evaluate the effectiveness of strategies used by these young people. The question that arises is whether in the context of lower differentiation between coping strategies used by boys with diabetes and those employed by girls, their coping efficiency is similar to that achieved by healthy boys.

The most significant results obtained in this study regard the relationship between coping styles in mothers and coping styles used by adolescents with diabetes. They show that emotion-oriented coping in mothers is negatively related to adolescents using the strategy of focusing on solving the problem. This emphasizes the need to support parents of teenagers with diabetes, since the stress they experience and coping strategies they employ may affect their children's adjustment. Thus, clinical intervention should include support for parents in developing their skills of effectively dealing with stress.

Conflicts of interest: No conflicts of interests were declared by the authors in relation to this article.

\section{REFERENCES}

Berg CA, Skinner M, Ko K, Butner J, Palmer DL, Wiebe DJ. The fit between stress appraisal and dyadic coping in understanding perceived coping effectiveness for adolescents with Type 1 diabetes. J Fam Psychol 2009; 23: 521 30 .

Beveridge RM, Berg CA, Wiebe DJ, Palmer DL. Mother and adolescent representations of illness ownership and stressful events surrounding diabetes. J Pediatrics Psychol 2006; 31: 818-27.

Dabrowska A, Pisula E. Parenting stress and coping styles in mothers and fathers of pre-school children with autism and Down syndrome. J Intellectual Dis Res 2010; 54: 266-80.

Endler N S, Parker J D A. Coping Inventory for Stressful Situation. Manual. Toronto, Multi-Health Systems, 1990.

Frydenberg E, Lewis R. How adolescents cope with different concerns: the development of the Adolescent Coping Checklist (ACC). Psychol Test Bull 1990; 3: 63-73.

Frydenberg E, Lewis R. Relations among well-being, avoidant coping, and active coping in a large sample of Australian adolescents. Psychol Rep 2009; 104: 745-58.

Graue M, Wentzel-Larsen T, Bru E, Hanestad BR, Sdegreesvik $\mathrm{O}$. The coping styles of adolescents with Type 1 diabetes are associated with degree of metabolic control. Diabetes Care 2004; 27: 1313-17.

Hema DA, Roper SO, Nehring JW, Call A, Mandleco BL, Dyches TT. Daily stressors and coping responses of children and adolescents with Type 1 diabetes. Child Care Health Dev 2009; 35: 330-39.

Hoff AL, Mullins LL, Gillaspy SR, Page MC, Van Pelt JC, Chaney JM. An intervention to decrease uncertainty and distress among parents of children newly diagnosed with diabetes: A pilot study. Families Syst Health 2005; 23: 329-42.

Huus K, Enskär K. Adolescents' experience of living with diabetes. Paediatr Nurs 2007; 19: 29-31.

Malik JA, Koot H M. Explaining the adjustment of adolescents with Type 1 diabetes. Diabetes Care 2009; 32: 774 9.

Mullins LL, Fuemeller BE, Hoff A, Chaney JM, Van Pelt J, Ewing CA. The relationship of parental overprotection and perceived child vulnerability to depressive symptomatology in children with Type 1 diabetes mellitus: The moderating influence of parenting stress. Children's Health Care 2004; 33: 21-34.

Olsen B, Berg CA, Wiebe DJ. Dissimilarity in mother and adolescent illness representations of Type 1 diabetes and negative emotional adjustment. Psychol Health 2008; 23: 113-29.

Olson AL, Johansen SG, Powers LE, Pope JB, Klein RB. Cognitive coping strategies of children with chronic illness. J Dev Behav Pediatr 1993; 14: 217-23.

Petersen C, Schmidt S, Bullinger M, DISABKIDS Group. Coping with a chronic pediatric health condition and health-related quality of life. Eur Psychologist 2006; 11: 50-6.

Pisula E, Sikora R. Age, gender and coping with stress by adolescents aged 12-17 years. Przeglad Psychologiczny 2008; 51: 405-21 (Article in Polish).

Seiffge-Krenke I. Diabetic Adolescents And Their Families: Stress, Coping, And Adaptation. New York, Cambridge University Press, 2001.

Shur H, Gamsu D, Barley V. The young person's perspective on living and coping with diabetes. J Health Psychol 1999; 4: 223-36.

Sikora R, Pisula E. Causes of stress and coping strategies in young people aged 14-16 years. Polskie Forum Psychologiczne 2002; 2: 110-22 (Article in Polish).

Strelau J, Jaworowska A, Wrzesniewski K, Szczepaniak P. Coping Inventory for Stressful Situations (Polish adaptation, manual). Warsaw, Polish Psychological Society, 2005.

Address for correspondence:

Ewa Pisula

Faculty of Psychology

University of Warsaw

5/7, Stawki, St.

00-183 Warsaw

Poland

Phone: +48 225549785

E-mail: ewa.pisula@psych.uw.edu.pl 